IN BRIEF

PROTEOMICS

Membrane protein interactome profiling

Carlson, M. L. et al. elife 8, e46615 (2019).

Co-fractionation combined with quantitative proteomics has become a standard highthroughput technique for profiling soluble protein interactions. Membrane protein interactions, however, are more difficult to capture due to the use of detergents that weaken their interactions and stability. Carlson et al. describe a method that utilizes peptidisc, a membrane protein mimetic, to trap a bacterial cell membrane proteome into watersoluble particles. These are then fractionated, isotopically labeled and characterized using mass spectrometry. In Escherichia coli, they were able to identify 4,900 binary interactions; previously wellcharacterized systems, such as SecY translocon, the Bam complex and the MetNI transporter, showed that many transient interactions were captured. Novel discoveries included a transperiplasmic supercomplex comprised of subunits from the Bam and Sec complexes along with membrane-bound chaperones, new interactors of the Bam complex, and identification of a novel type I ABC transporter.

https://doi.org/10.1038/s41592-019-0601-x

STEM CELLS

\section{Making limb-like structures from mouse PSCs}

Mori, S. et al. Nat. Commun. 10, 3802 (2019).

Organoids can serve as a proxy for developmental processes such as organogenesis. While a variety of different tissues have been modelled with organoids, the limb bud has not been one of them. Mori et al. adapted the SFEBq (serum-free floating culture of embryoid body-like aggregates with quick reaggregation) approach. They found that culturing aggregates of mouse embryonic pluripotent stem cells (PSCs) in the presence of Matrigel and BMP4 induces hindlimb bud-like tissue, consisting of an outer epithelial layer with aggregated cells internally. Retinoic acid promotes forelimb bud fate, while retinoic acid antagonists favor a hindlimb bud fate. Moreover, if BMP was provided in a locally restricted manner, the epithelium locally thickened in response, reminiscent of dorsoventral patterning. The developed protocol, therefore, mimics early stages of appendage formation, while further adjustments are needed to enable distal elongation as in the developing mouse embryonic limb.

\section{NEUROSCIENCE}

\section{Tracing neurons made easy \\ Wang, Y. et al. Nat. Commun. 10, 3474 (2019).}

Neuronal morphology is a key attribute for distinguishing neurons and determining their cell type. Yet, tracing neurons in light-microscopic image stacks remains an arduous process. Wang et al. facilitate this task by incorporating virtual reality (VR) into the workflow. Their TeraVR platform is based on, and extends, the TeraFly tool for handling large image datasets. TeraVR facilitates tracing neurons in difficult conditions such as at low signal-to-noise ratios. The platform also allows faster tracing speeds than alternative software tools. An attractive feature of TeraVR is that multiple users can work on a particular neuron reconstruction together through the cloud, even if they are located in different cities. TeraVR is available as part of Vaa3D via https://github.com/Vaa3D.

https://doi.org/10.1038/s41592-019-0602-9

\section{IMAGING \\ Improving optical \\ coherence tomography}

Zhou, K.C. et al. Nat. Photon. http://doi.org/dbgq (2019).

Optical coherence tomography (OCT) is a label-free imaging technique based on low-coherence interferometry, and is widely used for tissue-imaging and in clinical applications. Long depth-of-focus OCT, which is used for imaging at depths within tissues, often has anistropic resolution, with lateral resolution being worse than axial resolution. In addition, conventional OCT images can be distorted due to refractive index inhomogeneity within samples. To overcome these limitations, Zhou et al. introduce optical coherence refraction tomography (OCRT). OCRT uses multiple OCT cross-sectional images, taken at diverse angles, to reconstruct high-resolution images. This works by extending the axial coherence gating that confers high axial resolution to the lateral dimension, and allowing differently distorted cross-sectional images to be de-warped and registered for correction. The researchers demonstrated the benefits of the approach by imaging several mouse organs as well as the human cornea.
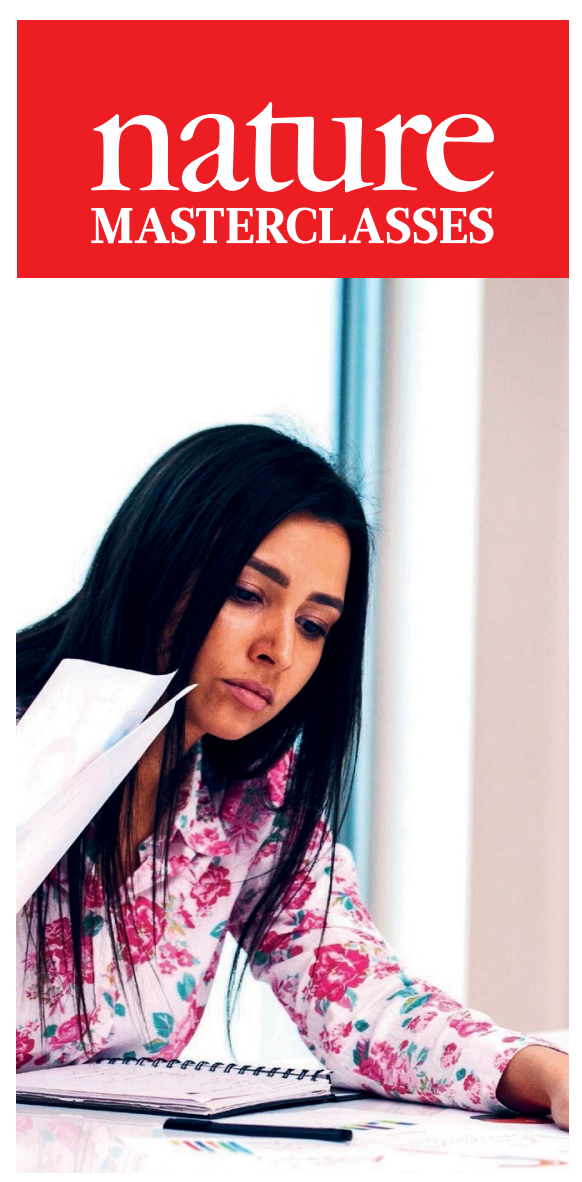

\section{Free online}

course for peer reviewers

\section{For researchers new to \\ peer review or wishing \\ to develop their skills}

\section{Register for free access at masterclasses.nature.com}

Taught by Nature Research editors • 3-4 hours' learning • Free completion certificate 